\title{
Black hole origins of active nuclei challenged in Armenia
}

\section{from Martin Ward and Ian Robson}

The Byurakan Observatory in Soviet Armenia has made outstanding contributions to the study of active galactic nuclei. Over the past 20 years Victor Markarian (who died late last year) and co-workers have identified more than 1,500 celestial objects with ultraviolet excess. This ensemble includes many examples from the active galactic zoo - starbursts, Seyfert galaxies and quasars - so the observatory provided a natural focus for a meeting*. The conference encompassed the study of nearly normal galaxies to the super energetic quasars - - a range in energy output of more than a million - but some general themes emerged: for example, is material being pulled into or thrown out of the nucleus?

E. Khachikian reminded us that one of the first people to suggest that ejection of material plays a major role in galactic nuclei was academician Ambartsumian. The question of ejection from, and accretion onto, active nuclei has proved rather complex. It is well established that radio jets are associated with ejection, whereas the interpretation of optical evidence is less clear. Optical filamentary structures or jets have been seen and in some cases they strongly suggest the outflow of gas from the nucleus. On the other hand, the inflow of material is also indicated. The famous active galaxy NGC 1275 has a system of approximately radial filaments which are believed to result from cooling gas falling toward the gravitational centre of the galaxy. It is possible that this phenomenon may be generalized to explain the weak optical emission lines seen in the nuclei of elliptical and SO galaxies. Elaine Sadler (Kitt Peak National Observatory) showed that in cases where there are strong optical emission lines a correlation exists with the nuclear radio emission. In Seyfert nuclei the bulk motion of some of the high density ionized gas may exceed the escape velocity of the central massive object, while other material, further from the nucleus, may lose angular momentum and move inwards. This originally distant matter is eventually accreted by the nucleus to provide fuel for the ultimate energy source powering the activity.

The once controversial subject of quasar redshifts - a cosmological versus an intrinsic or local origin - is no longer an issuc. As the conference demonstrated, the evidence in favour of the cosmological interpretation is now over-

* Observational Evidences for the Activity in the Nuclei of Galaxies IAU Symposium 121. Byurakan, Armenia, 3-7 June 1986. whelming in the view of the vast majority of astronomers. Reviews by Howard Yee (University of Montreal) and John Hutchings (Dominion Astrophysical Observatory) showed that the argument has shifted to discussion of what sort of host galaxy a quasar resides within, spiral or elliptical, and whether interactions or other morphological disturbances are a common feature in the nearby examples. Here, imaging with the sensitive chargecoupled device detectors has recently much strengthened the association between quasars and parent galaxies as well as indicating that they may be found preferentially in groups of galaxies. The effects of the powerful radiation field of quasars on the interstellar material in the parent galaxy may be sufficient to light up the entire galaxy, resulting in observable spatially extended emission lines.

A new controversy concerning active nuclei has emerged over the past few years. Roberto Terlevich (Royal Greenwich Observatory) and Jorge Melnick (European Southern Observatory) propose to remove the need for the presence of exotic objects (black holes) at the centres of at least some active galaxies and replace them with superhot, massive stars which they claim are produced naturally as a result of simple stellar evolution. It turns out that the part of the continuum emission that ionizes the gas is similar to that predicted for the radiation processes associated with the black hole models. Thus, the observed emission line strengths can be matched by both models. Furthermore, the high supernovae rate that is a consequence of the hot star hypothesis can reproduce the emission line characteristics of the more energetic Seyfert galaxies. Even the collimated radio structures observed might be produced from confined stellar winds and bipolar flow. Those favouring the presence of exotic objects have pointed to the highest luminosity quasars, which would be difficult to explain by thermal processes. Also, the very short-term quasi-periodic $\mathrm{X}$-ray variations in several of the lower luminosity Seyferts are more likely to be understood in terms of an accretion process.

No-one would argue that ionization by hot stars takes place in so-called starburst nuclei. Nor that in some cases the regions around Seyfert nuclei are dominated by a young stellar population. The argument really concerns how general is the stellar origin of the ionizing continuum. The field is ripe for observational tests and some are already under way.

As a postscript to the conference a few of the participants visited the Soviet 6metre optical telescope. This, the world's largest instrument, is now equipped with an image photon counting system for spectroscopy. In the past Soviet astronomers have sometimes been justifiably peeved by their western counterparts' apparent disinclination to reference or take full notice of some areas of their research. This situation is likely to change, as the technological gap in the field appears to be narrowing.

Martin Ward is in the Astronomy Department of the University of Washington, Seattle 98195 , USA; Ian Robson is in the School of Physics and Astronomy, Lancashire Polytechnic, Preston PR1 2TQ, UK.

\section{Fast glacier flow}

\section{A soft bed is not the whole answer}

\section{from Gordon de Q. Robin}

NEw evidence of a few metres of soft deforming glacial till beneath the low slopes but fast flow of Ice Stream B in West Antarctica ${ }^{1.2}$ led Boulton ${ }^{3}$ in a recent News and Views article to renew his call for a shift of emphasis in the study of ice sheets, past and present. He considers that glaciers find sliding over rock both harder and slower than motion over deformable till, and that such easy motion over till was common among former midlatitude ice sheets. Although primarily directed at effects of future climatic change on ice sheets, a recent conference was relevant to Boulton's plea and brought together evidence and ideas on the flow of three types of glaciers: ice streams; surging glaciers whose fast flow is intermittent; and tidewater glaciers whose fast flow leads to their discharge into the sea*.

What is the difficulty in understanding fast glacier flow? Almost certainly such flow implies 'sliding', but to many glaciologists the term covers both sliding of clean ice over hard bedrock and/or the rapid deformation of a basal layer up to a few metres thick, be it of ice, ice-moraine mixture or water-saturated moraine. Theoretical studies of glacier flow over such a deforming boundary layer are available $e^{4,5}$ but the problem is to define the

*The American Geophysical Union Chapman Conference, Whistler, British Columbia, 4-8 May 1986. 